\title{
What is pulmonary arterial hypertension?
}

Pulmonary arterial hypertension (PAH) is a term that was coined in 1998 at the World Health Organization Symposium on Pulmonary Hypertension at Evian, France. Because a clinically useful classification for pulmonary hypertension was lacking, the late Alfred Fishman proposed a clinical classification system for pulmonary hypertension which has been widely adopted. ${ }^{[1]}$ Its purpose was to provide a guide to physicians who encounter a patient with pulmonary hypertension of uncertain etiology. The classification system helped direct the clinical evaluation of the patient so that underlying disease(s) that might be causing or contributing to the pulmonary hypertension could be identified. PAH (also known as Category 1) includes patients who have an elevation in pulmonary artery pressure with a normal pulmonary wedge pressure, either alone or in association with many other diseases.

In 1995, intravenous epoprostenol became the first treatment approved by the FDA for PPH (now referred to as IPAH). ${ }^{[2]}$ Subsequently, a second epoprostenol trial for patients with pulmonary hypertension associated with scleroderma resulted in FDA approval for that condition. ${ }^{[3]}$ Both studies were initiated prior to the Evian classification system. In 2000, the FDA approved bosentan for pulmonary hypertension, but in this instance the FDA agreed to approve it for the entire category of pulmonary hypertension known as PAH (Category 1) and did not require separate clinical trials to evaluate its efficacy in patients with different associated diseases. ${ }^{[4]}$ The reason behind the FDA's decision to do this has not been disclosed. However, since then, all of the new therapies (prostacyclins, endothelin receptor blockers, and phosphodiesterase-5 inhibitors) that have been approved by the FDA to treat patients with pulmonary hypertension have been approved for the entire category of PAH.$^{[5]}$ As a result, physicians are left with the perception that these therapies are safe and effective for patients with Category $1 \mathrm{PAH}$ irrespective of the associated condition, and are neither safe nor effective for patients with pulmonary hypertension that fall into the other four categories. Whether or not this is true is unknown but it has wide implications for those patients whose pulmonary hypertension falls outside of Category 1.

Implicit with the designation of $\mathrm{PAH}$ is the notion that the disease originates and/or is confined to the pulmonary arteriolar bed, and that these drugs in some way target that region of the pulmonary circulation. ${ }^{[5]}$ Yet there has never been a human clinical trial that has demonstrated the mechanism or site of action of any of these approved therapies on the pulmonary circulation in patients with $\mathrm{PAH}$. We do not know if prostacyclin receptors in the lung are stimulated, whether endothelin receptors in the lung are blocked, or whether phosphodiesterase- 5 is inhibited in the pulmonary arteriole. We do know that patients who are treated with any therapy walk farther with a 6-Minute Walk Test than those who are not. ${ }^{[6]}$

If the target of these treatments is the pulmonary arteriole, I ask why other patients whose disease is similar (namely have severe elevations in PA pressure associated with RV failure and reduced exercise tolerance) are not treated the same. As the Figure 1 shown demonstrates, arteriolar medial hypertrophy and intimal proliferation can occur in patients with pulmonary hypertension that fall into Categories 2, 3, and 4, as well. ${ }^{[7]}$ The case has been made that "severe" pulmonary hypertension is distinguished from "mild" pulmonary hypertension based on the nature of the vascular changes rather than from the etiology. ${ }^{[8]}$

At the present time, there is keen interest in developing more effective treatments for patients with pulmonary hypertension that will be disease modifying, with the ability to halt the progression, or induce regression of the pulmonary vascular
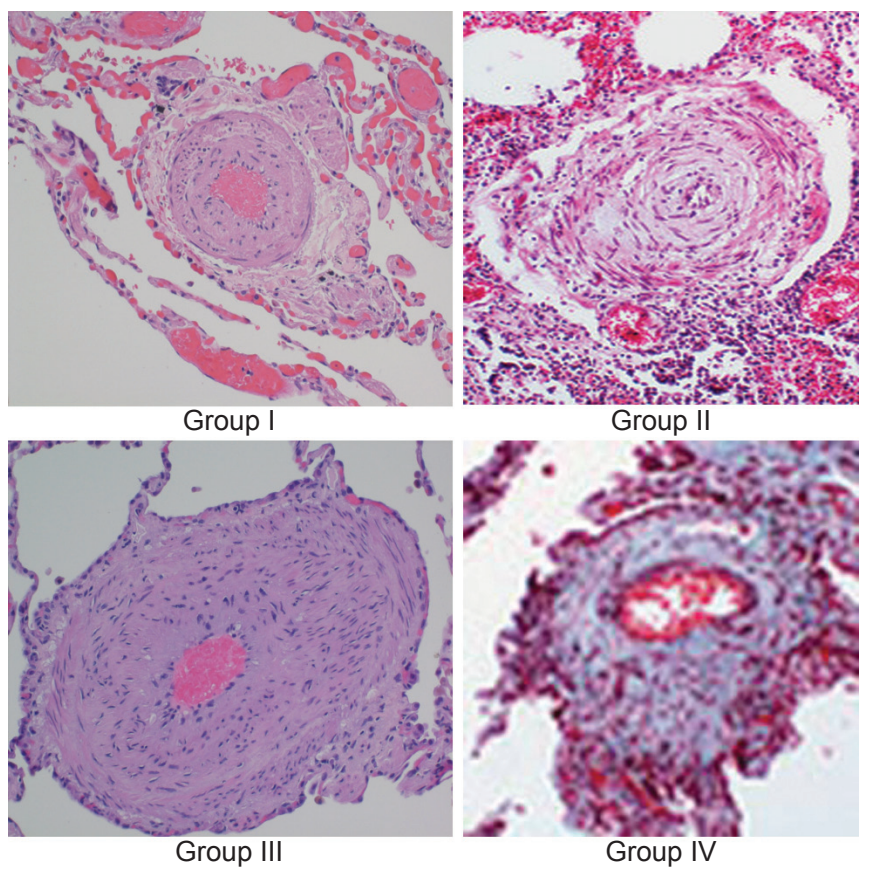

Figure 1: The pulmonary vasculature in lung tissue from four patients with severe pulmonary hypertension is shown. The patients were characterized clinically as Category 1 (IPAH), Category 2 (left ventricular failure), Category 3 (interstitial lung disease), and Category 4 (chronic thromboembolic disease). However, each specimen reveals similar changes in the pulmonary arteriole showing medial hypertrophy and intimal proliferation. Without knowing the clinical phenotype it would not be possible to distinguish them based on the vascular pathology. 
disease. As we broaden our vision to include a wide spectrum of treatments that may interact with several molecular pathways that are involved in $\mathrm{PAH}$, we would do well to also broaden our vision to include the spectrum of patients that have severe pulmonary hypertension, whether in association with congenital heart disease, connective tissue disease, left ventricular heart failure, parenchymal lung disease, or thromboembolic disease. Clinical trials can be designed to identify the characteristics of patients who respond and those who do not, and the influence of comorbid diseases on drug efficacy and safety. ${ }^{[9]}$ And while I am not predicting that the treatments will be successful across all etiologies, I question why we are ignoring the larger group of patients who, in spite of their similar hemodynamics and pulmonary vascular pathology, are excluded because of a classification system that was never intended to direct therapeutic decisions.

\section{Stuart Rich}

Section of Cardiology, University of Chicago, Chicago, IL, USA Email: srich@medicine.bsd.uchicago.edu

\section{REFERENCES}

1. Rich S. Classification of pulmonary hypertension. In: Pulmonary Circulation. 2nd ed. In: Peacock AJ, Rubin LJ, editors. London: Edward Arnold Ltd.; 2004. p. 147-54.
2. Barst RJ, Rubin LF, Long WA, McGoon MD, Rich S, Badesch DB, et al A comparison of continuous intravenous epoprostenol (prostacyclin) with conventional therapy for primary pulmonary hypertension. New Engl J Med 1996;334:296-301.

3. Badesch DB, Tapson VF, McGoon MD, Brundage BH, Rubin LJ, Wigley FM, et al. Continuous intravenous epoprostenol for pulmonary hypertension due to the scleroderma spectrum of disease: A randomized, controlled trial. Ann Intern Med 2000;132:425-34.

4. Rubin LJ, Badesch DB, Barst RJ, Galiè N, Black CM, Keogh A, et al Bosentan therapy for pulmonary arterial hypertension. N Engl J Med 2002;346:896-903.

5. Humbert M, Sitbon O, Simonneau G. Treatment of Pulmonary Arterial Hypertension. N Engl J Med 2004;351:1425-36.

6. Macchia A, Marchioli R, Tognoni G, Scarano M, Marfisi RM, Tavazzi L, et al. Systematic review of trials using vasodilators in pulmonary arterial hypertension: why a new approach is needed. Am Heart J 2009;159:245-57.

7. Rich S, Rabinovitch M. The diagnosis and treatment of secondary (non-category 1) pulmonary hypertension. Circulation 2008;118:2190-9.

8. Voelkel NF, Tuder RM. Hypoxia induced pulmonary vascular remodeling: A model for what human disease? J Clin Invest 2000;106:733-8.

9. Mehta C, Gao P, Bhatt DL, Harrington RA, Skerjanec S, Ware JH Statistical primer for cardiovascular research: Optimizing trial design: Sequential, adaptive, and enrichment strategies. Circulation 2009;119: 597-605.

\begin{tabular}{|l|l|}
\hline \multicolumn{2}{|c|}{ Access this article online } \\
\hline Quick Response Code: & Website: www.pulmonarycirculation.org \\
\hline & DOI: 10.4103/2045-8932.101388 \\
\cline { 2 - 2 } & \\
& $\begin{array}{l}\text { How to cite this article: Rich S. What is } \\
\text { pulmonary arterial hypertension? Pulm Circ } \\
\text { 2012;2:271-2. }\end{array}$ \\
\end{tabular}

